\title{
Improving care for patients after transient ischaemic attack (TIA)
}

\section{Anthony G Rudd}

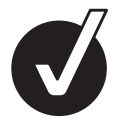

There is no doubt that progress has been made in the care of patients with transient EDITOR'S ischaemic attack (TIA) over CHOICE recent years. Virtually all hospitals now offer rapid access neurovascular clinics or admission for high risk patients with many units running clinics 7 days a week. Availability of brain and carotid imaging has improved ${ }^{1}$ and the UK Carotid Interventions Audit has shown dramatic improvements in the symptom to surgery times coming down from a median of 28 days in 2008 to 21 days in 2010 and 14 days in $2012 .^{2}$ However, in this edition of the journal Lager et al report the results of a study examining how effectively risk factors are managed in primary care in the UK after a diagnosis of TIA has been made in a specialist clinic. ${ }^{3}$ The key message is that substantial improvements are needed if we are to minimise the risk of further cerebrovascular disease in all patients. And it is worth pointing out that it is not just stroke that can be avoided but vascular dementia, ischaemic heart disease and peripheral vascular disease as well. There is no shortage of good papers and policy documents that define the standards that we should be achieving. ${ }^{4-6}$ We know that the risk of stroke in the first month after stroke is substantial particularly for those with hemispheric symptoms, older patients, those with hypertension, diabetes and where the symptoms are prolonged $^{7}$ and we know that rapid management can substantially reduce the risk of stroke. ${ }^{8}$ Most guidelines recommend that patients with high risk TIA $\left(\mathrm{ABCD}^{2} 4\right.$ or more) should be seen within $24 \mathrm{~h}$ of symptoms and those with a lower risk within a maximum of 7 days. This dichotomisation of patients into low and high risk groups was developed as a way of reducing the burden on emergency services, although it does not really make much clinical or managerial sense. Even the low risk patients are at a risk of stroke within the first week and if a clinic is

Correspondence to Professor Anthony G Rudd, Kings College London, Stroke Unit, St Thomas' NHS Foundation Trust, Westminster Bridge Rd, London SE1 7EH, UK; anthony.rudd@kcl.ac.uk available to see patients within $24 \mathrm{~h}$ it may as well see all patients. What seems more difficult to achieve is to get patients to take TIA seriously and treat it as a medical emergency. Interviews with 1000 patients in Oxfordshire who had had a minor stroke or TIA between 2002 and 2007 showed that $70 \%$ of patients do not correctly recognise their TIA or minor stroke and $30 \%$ delay seeking medical attention for more than $24 \mathrm{~h}$. Nearly a third of recurrent strokes occur before the patient seeks medical attention. ${ }^{9}$ A systematic review of delays seeking medical attention with data from nine different studies from around the world showed significant variability between studies, but all showing many patients delaying seeking medical attention regardless of whether the patient accurately recognised the cause of their symptoms. ${ }^{10}$ A study of the influence of general practice opening hours showed that patients are more likely to present promptly if their symptoms occur during normal working hours. ${ }^{11}$ So, we need somehow to educate the population as to what the symptoms of stroke and TIA are and emphasise the importance of seeking specialist help urgently. Patients often appear reluctant to bother emergency services when the symptoms have resolved as they usually have after TIA. Reducing the bureaucracy of obtaining specialist medical care could help. Enabling paramedics to refer directly to a neurovascular clinic and having an open access walk in clinics might reduce delays in delivering the necessary care.

There is less information about the level of knowledge of TIA and minor stroke by professionals in primary care and the emergency departments; however, a rather disturbing questionnaire based study found that while most general practitioners would prescribe antiplatelet therapy, $22 \%-40 \%$ would not refer first time TIA patients, depending upon the presenting symptom. ${ }^{12}$ Anecdotal reports and certainly my experience is that the quality of diagnosis of TIA in primary care is inadequate. Less than $50 \%$ of referrals to neurovascular clinics actually have cerebrovascular disease. A study from Toronto $^{13}$ shows that outcomes for patients who are referred to a stroke prevention clinic are better than those who are not with a $26 \%$ reduction in 1-year mortality (HR, 0.74; 95\% CI 0.65 to 0.84 ). This was not a randomised controlled study, although the authors did attempt to control for the key variables and there may well have been significant bias. Nevertheless, combined with the EXPRESS study ${ }^{8}$ these data provide a strong pointer to the advantages of rapid specialist input following TIA or minor stroke. Patients suspected of having TIA should be started immediately on an antiplatelet agent by the first clinician who sees them. This means the drug should be swallowed straightaway. It is not adequate to put the prescription in the pocket of the patient to be collected later. Whether the drug used is aspirin or clopidogrel probably does not matter.

The next issue is how to ensure that patients continue to take their secondary prevention medication. In the USA, a cohort of over 2800 patients from 106 hospitals were followed up at 3 months ${ }^{14}$ and 1 year ${ }^{15}$ to assess adherence. By 3 months, a quarter had discontinued some of their medications and by a year this had risen to a third. Independent predictors of persisting with medication were fewer drugs prescribed at onset, an adequate income, having an appointment with a primary care provider and better understanding of what the drugs were for and their side effects. The targets for lipid and blood pressure control given in the National Clinical Guidelines for Stroke ${ }^{16}$ are more rigorous than those required by the quality outcomes framework (QOF) and are the ones that should be achieved if maximum benefit is to be obtained from prevention treatment. The QOF has been a highly successful initiative that has resulted in major improvements in care but it is time that the standards for stroke secondary prevention were reviewed to come into line with the evidence.

We have the structures in place in the UK to deliver high quality care for patients with TIA. What is lacking is sufficient understanding of the disease by the public and some professionals working in primary care and insufficiently rigorous QOF targets for secondary prevention. Addressing these issues is where resources should now be directed through public awareness campaigns and better education of health professionals.

Contributors AGR was responsible for researching and writing the editorial.

Competing interests None. 
Provenance and peer review Commissioned; internally peer reviewed.

Published Online First 25 April 2012

Postgrad Med J 2012;88:303-304.

doi:10.1136/postgradmedj-2012-130883

\section{REFERENCES}

1. Intercollegiate Stroke Working Party. National Sentinel Audit of Stroke Royal College of Physicians London, 2011. http://www.rcplondon.ac.uk/stroke

2. Carotid Interventions Audit 4th Round Public Report. 2012. http://www.rcplondon.ac.uk/resources/ukcarotid-interventions-audit

3. Lager KE, Wilson A, Khunti K, et al. Quality of secondary prevention measures in TIA patients: a retrospective cohort study. Postgrad Med J 2012:88:305-11.

4. Rothwell PM, Algra A, Amarenco P. Medical treatment in acute and long-term secondary prevention after transient ischaemic attack and ischaemic stroke. Lancet 2011;377:1681-92.

5. NICE guideline on Diagnosis And Initial Management of Acute Stroke and TIA. London, UK: Royal College of Physicians, 2008.
6. Department of Health. National Stroke Strategy. London, 2007. http://www.dh.gov.uk/publications

7. Giles MF, Rothwell PM. Systematic review and pooled analysis of published and unpublished validations of the $A B C D$ and $A B C D^{2}$ transient ischaemic attack risk scores. Stroke 2010:41:667-73.

8. Rothwell PM, Giles MF, Chandratheva A, et al; Early use of Existing Preventive Strategies for Stroke (EXPRESS) study. Effect of urgent treatment of transient ischaemic attack and minor stroke on early recurrent stroke (EXPRESS study): a prospective population-based sequential comparison. Lancet 2007:370:1432-42.

9. Chandratheva A, Lasserson DS, Geraghty OC, et al Oxford Vascular StudyPopulation-based study of behavior immediately after transient ischemic attack and minor stroke in 1000 consecutive patients: lessons for public education. Stroke 2010;41:1108-14

10. Sprigg N, Machili C, Otter ME, et al. A systematic review of delays in seeking medical attention after transient ischaemic attack. J Neurol Neurosurg Psychiatry 2009;80:871-5.

11. Lasserson DS, Chandratheva A, Giles MF, et al. Influence of general practice opening hours on delay in seeking medical attention after transient ischaemic attack (TIA) and minor stroke: prospective population based study. BMJ 2008;337:a1569.

12. Jagadesham VP, Aparajita R, Gough MJ. Can the UK guidelines for stroke be effective? Attitudes to the symptoms of a transient ischaemic attack among the general public and doctors. Clin Med 2008; 8:366-70

13. Webster F, Saposnik G, Kapral MK, et al. Organized outpatient care: stroke prevention clinic referrals are associated with reduced mortality after transient ischemic attack and ischemic stroke. Stroke 2011;42:3176-82.

14. Bushnell CD, Zimmer LO, Pan W, et al. Adherence evaluation after ischemic stroke-longitudinal investigators persistence with stroke prevention medications 3 months after hospitalization. Arch Neurol 2010;67:1456-63.

15. Bushnell CD, Olson DM, Zhao X, et al. AVAll Investigators secondary preventive medication persistence and adherence 1 year after stroke. Neurology 2011;77:1182-90.

16. National Clinical Guidelines for Stroke. 3rd edn London: Intercollegiate stroke working Party, Royal College Physicians, 2008. http://bookshop.rcplondon ac.uk/contents/6ad05aab-8400-494c-8cf49772d1d5301b.pd 